University of Nebraska - Lincoln

DigitalCommons@University of Nebraska - Lincoln

Timothy J. Gay Publications

Research Papers in Physics and Astronomy

$5-2008$

\title{
Active feedback scheme for minimization of helicity-dependent instrumental asymmetries
}

\author{
M. I. Fabrikant \\ University of Nebraska - Lincoln, ifayam@gmail.com \\ K. W. Trantham \\ Fort Hays State University \\ V. M. Andrianarijaona \\ Pacific Union College \\ Timothy J. Gay \\ University of Nebraska - Lincoln, tgay1@unl.edu
}

Follow this and additional works at: https://digitalcommons.unl.edu/physicsgay

Part of the Physics Commons

Fabrikant, M. I.; Trantham, K. W.; Andrianarijaona, V. M.; and Gay, Timothy J., "Active feedback scheme for minimization of helicity-dependent instrumental asymmetries" (2008). Timothy J. Gay Publications. 57. https://digitalcommons.unl.edu/physicsgay/57

This Article is brought to you for free and open access by the Research Papers in Physics and Astronomy at DigitalCommons@University of Nebraska - Lincoln. It has been accepted for inclusion in Timothy J. Gay Publications by an authorized administrator of DigitalCommons@University of Nebraska - Lincoln. 


\title{
Active feedback scheme for minimization of helicity-dependent instrumental asymmetries
}

\author{
M. I. Fabrikant, ${ }^{1}$ K. W. Trantham, ${ }^{2}$ V. M. Andrianarijaona, ${ }^{3}$ and T. J. Gay ${ }^{1}$ \\ 1. Behlen Laboratory of Physics, University of Nebraska-Lincoln, \\ Lincoln, Nebraska 68588, USA \\ 2. Fort Hays State University, Hays, Kansas 67601, USA \\ 3. Pacific Union College, Angwin, California 94508, USA \\ Corresponding author - M. I. Fabrikant, ifayam@gmail.com
}

\begin{abstract}
A method for the active feedback reduction of optical instrumental intensity asymmetries is presented. It is based on the fast chopping of two spatially separated beams of light with orthogonal linear polarizations that are recombined and passed through a quarter-wave plate to yield a single beam with rapidly flipping helicity. Active electro-optic feedback has been successfully employed to maintain this asymmetry below $10^{-5}$.
\end{abstract}

\section{Introduction}

Of the available methods to produce polarized electrons, laser-induced photoemission from GaAs crystals is advantageous because it allows the rapid flipping of electron spin using optical means [1]. Weaknesses of this method emerge if the experimental signal is sensitive to spin; helicity reversal of the laser beam is generally associated with both spatial and intensity variations, which are produced by the optical elements responsible for helicity reversal. If the GaAs crystal has a nonuniform quantum efficiency over its surface, helicity-related spatial movement of the laser beam may produce a helicity-dependent current asymmetry emerging from the polarized electron source. Similarly a helicity-dependent laser intensity will produce a corresponding helicity-dependent current, even in the absence of spatial variations. Polarized electron currents that depend on their spin direction will subsequently cause an instrumental asymmetry (IA) mimicking a true spin-dependent signal. In this paper we define photon intensity or electron current asymmetry as

$$
A=\frac{I^{-}-I^{+}}{I^{-}+I^{+}}
$$

where $I^{+}$is the current or intensity of predominantly forward-spin electrons or photons and $I^{-}$is the equivalent quantity for spin-backward particles. We present an active feedback scheme to correct this problem at the optical level. In previous work [2] we have shown that the upper limit of IA with passively stabilized optical spin reversal is $\sim 5 \times 10^{-4}$. Active feedback to correct this problem has been investigated by several groups [3-8], but to our knowledge this is the first scheme that does not make use of electro-optic or electromechanical feedback operating at the helicity-reversal frequency to force instrumental asymmetries to zero.

The work reported here was necessitated by the requirements of a larger experiment designed to observe electron circular dichroism (ECD) $[9,10]$. In this experiment, polarized electrons with alternately forward and backward longitudinal spins traverse a chiral vapor target. The goal is to observe a spin-dependent asymmetry in the number of 
electrons that traverse the target. This asymmetry is expected to be very small - of the order of $10^{-4}$ [11]. In order to observe such small values, it is important that the experiment's IA be significantly smaller than $10^{-4}$.

\section{Apparatus}

Previous versions of our ECD experiment, which were plagued by large $\left(\sim 10^{-4}\right)$ IAs, employed passive measures such as temperature stabilization and refocusing optics to minimize these effects $[2,3]$. The helicity of the polarized light was flipped by using a single photoelastic modulator (PEM) to vary the retardance [12]. The primary source of instrumental asymmetries resulted from the spatial drifting of the light beam, which is an inherent consequence of the PEM $[2,13]$. To overcome these problems we devised an optical system that flips the helicity of polarized light mechanically rather than electro-optically and that uses slow active feedback to limit time-varying IAs.

The optical system is shown schematically in Figure 1. It uses a $75 \mathrm{~mW}, 785 \mathrm{~nm}$ Power Technology diode laser (Model LDCU5/7873). This laser was specially built to have excellent polarization and intensity stability with regard to temperature. Nevertheless we use a linear polarizer (LP) to ensure the initial light polarization. Afterward the laser light passes through a crystalline half-waveplate (Meadowlark H1557) and a computer-controlled liquid crystal retarder (LCR: Meadowlark LVR-200). It then passes through a polarizing beam splitter (Thorlabs PBS3) creating two orthogonally polarized spatially separated beams of light. When the half-waveplate is rotated, intensity is redistributed between these two beams. A mechanical chopper (Thorlabs MC1000A) is inserted so that, when it is positioned correctly, it allows only one beam to pass at a time. The purpose of the LCR will be explained in Section 3. After the chopper, each beam is reflected by a mirror into a second

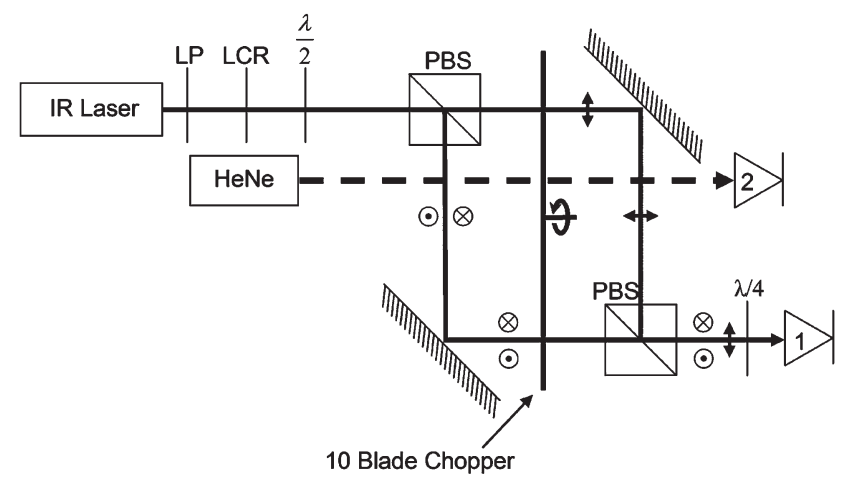

Figure 1. Schematic of the optical system. Light from the laser is separated into its constituent polarizations (arrows) and recombined in space, but not in time, with the aid of a chopper. The intensity of each polarization is measured with diode 1 (using the He-Ne signal from diode 2 as a reference) and the LCR is adjusted to minimize the intensity asymmetry. Helicity is flipped by the passage of the temporally alternating linear polarization through a fixed quarter-wave plate. polarizing beam splitter that acts to spatially recombine the two beams. The effect of this setup is to separate the beams in time using the chopper, but recombining them in space with the two beam splitters. The recombined beam then passes into diode 1 . The photodiode signal can be monitored with an oscilloscope or a computer. Finally a third beam, generated by a He-Ne laser, is shot through the chopper into diode 2 . This beam is used to create a reference signal with which we compare the signal that reaches diode 1. A synchronization output from the chopper controller was available, but was found not to remain in phase with the diode 1 signal.

\section{Measuring Asymmetry and Positive Feedback}

The problems associated with IAs lie not in their nonzero value per se, but in the fact that they drift in time. If an IA did not drift, it could be minimized by passive means, measured carefully, and subtracted from the ECD signal to yield true asymmetries. We consider first the problem of IA measurement, and then the use of active feedback to eliminate IA drifting.

The signals that reach diodes 1 and 2 are modified square waves [see Figures 2(a) and 2(b), respectively]. Both diode signals run to Keithly 1645 picoammeters, and the analog voltage outputs of these meters are sampled with a $\mathrm{Na}$ tional Instruments PCI-6143 data acquisition card. The card samples data in "burst mode" by making many measurements at the sampling frequency, storing them in a buffer,
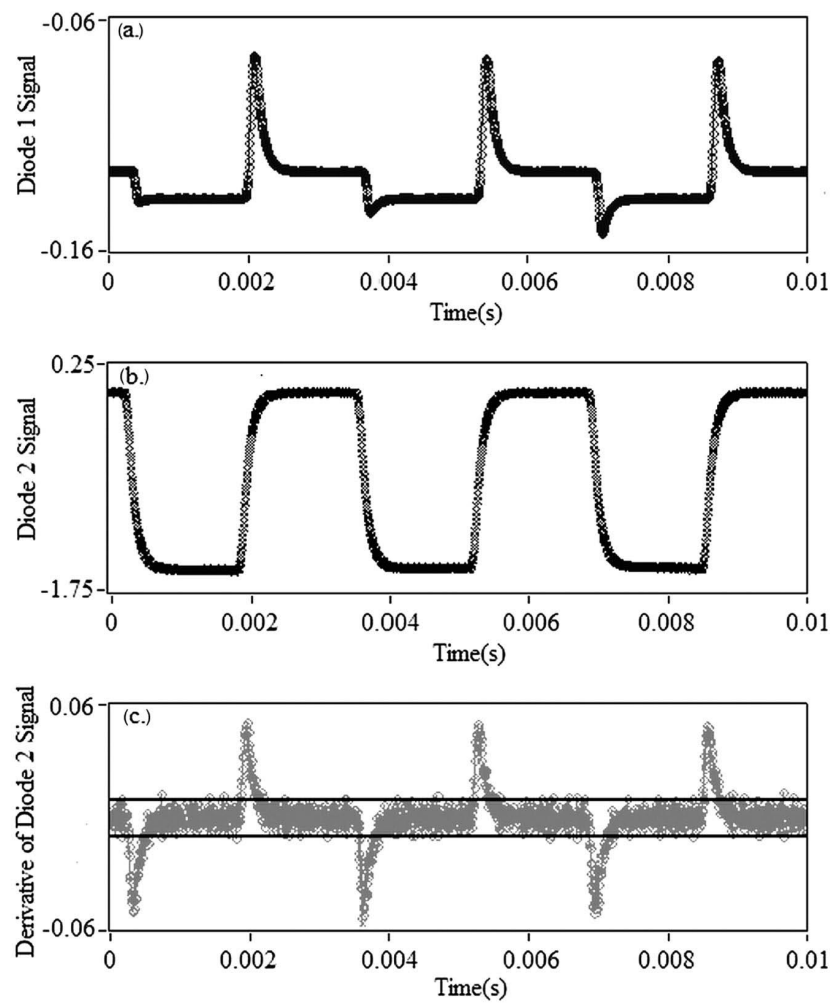

Figure 2. Raw signals in arbitrary units as read by the computer data acquisition card: (a) recombined intensity, (b) trigger waveform, (c) time derivative of the trigger signal with rejection thresholds indicated. 
and outputting all resulting data to the computer after the desired number of samples has been reached. The diode 1 signal represents the intensity of the recombined beams [see Figure 2(a)]. Since the separated beam intensities are generally different, a modified square-wave results. The spikes in the signal are the result of both beams being partially on simultaneously as the chopper switches from one blade to the next. The diode 2 signal is a square-wave that maintains a fixed phase relationship to the diode 1 signal.

Asymmetry $A$ can be calculated using $I^{+(-)}$taken from the upper (lower) flat regions of the square-wave in Figure 2(a). To get an accurate value for $I^{+}$and $I^{-}$, we must eliminate any sampling of data in the vicinity of the spikes in the square wave. We differentiate the reference signal [see Figure 2(c)] and reject all data points where the absolute slope of the trigger signal is less than some threshold - typically $0.01 \mathrm{~V} / \mathrm{s}$. We then use the trigger signal in combination with the chopping rate to bin all $I^{+}$and $I^{-}$points and take their respective averages. The region of the chopper cycle over which sampling occurs, as dictated by the reference beam, is shown in Figure 2(c).

The choice of chopper speed, sampling rate, and sampling time is dictated by three criteria: (1) at least one chopper waveform must be sampled, that is, a high and low intensity are required to calculate an intensity asymmetry; (2) the data acquisition time taken to measure an asymmetry value must be short compared to characteristic IA drift times; and (3) the chopping rate must not be faster than the response time of the diodes. A typical chopper frequency in these experiments was $500 \mathrm{~Hz}$ with a sample rate of $200 \mathrm{kHz}$ and a data acquisition time for one asymmetry value of $50 \mathrm{~ms}$.

We cannot assign an uncertainty to this value because there is no way of knowing whether these results obey a normal distribution or what the width of that distribution is. We therefore repeat this process 1000 times and extract a mean and standard deviation of the mean to give an average asymmetry measurement with an uncertainty. This compound measurement takes $\sim 1.2$ s to make. The number of data points used to determine a single asymmetry value is not taken into account explicitly when calculating an uncertainty for the composite asymmetry. Nevertheless as a consequence of the criteria above, this number influences the uncertainty and must be sufficiently high to allow a small uncertainty to be reached in a reasonable amount of time.

The main source of systematic error in any asymmetry measurement is asymmetry drift. One way to minimize this error is to chop the beam very quickly and sample many times in a given chopping period; the shorter the chopping period, the less opportunity the asymmetry has to drift within a given measurement of A. This improves the accuracy of any given asymmetry measurement but does not improve the problem of overall drift. To correct long-term drift, active feedback to set the IA to zero must be employed. An example of such an asymmetry measurement taken for our optical system over an extended period of time is shown in Figure 3. Over the course of $1.5 \mathrm{~h}$, the asymmetry drifts drastically to values much greater than the expected experimental asymmetry. To eliminate this drift we employ active feedback.

In the simplest system we would have only the halfwaveplate to orient the linear polarization precisely at $45^{\circ}$, just upstream of the first beam splitter. One way to feed back on asymmetry would be to make an asymmetry measurement and rotate the half-waveplate to continually force the asymmetry to zero. We can analyze the effects of various elements in our optical train using the technique of Mueller calculus [14]. In our case we begin with a vertically polarized state of light emitted by the laser:

$$
\vec{P}=\left[\begin{array}{r}
1 \\
-1 \\
0 \\
0
\end{array}\right]
$$

In this vector, $P_{0}$ is the total intensity, $P_{1}$ is the difference between the intensity of horizontally and vertically polarized light, $P_{2}$ is the intensity difference in the $45^{\circ}$ and $135^{\circ}$ polarization directions, and $P_{3}$ is the difference in intensity between right- and left-handed circularly polarized light. The half-waveplate can be represented by the following operator:

$$
\left[\frac{\lambda}{2}\right]=\left[\begin{array}{rrrr}
1 & 0 & 0 & 0 \\
0 & 1 & 0 & 0 \\
0 & 0 & -1 & 0 \\
0 & 0 & 0 & -1
\end{array}\right]
$$

To account for the rotation of the half-waveplate, we introduce the rotation operators

$$
\left[R_{ \pm}(a)\right]=\left[\begin{array}{rrrr}
1 & 0 & 0 & 0 \\
0 & \cos (2 a) & \mp \sin (2 a) & 0 \\
0 & \pm \sin (2 a) & \cos (2 a) & 0 \\
0 & 0 & 0 & 1
\end{array}\right]
$$

where $a$ is the angle of rotation. Multiplying these matrices together gives us a final Stokes vector:

$$
\vec{P}_{\text {final }}=\left[R_{a}\right] \cdot[\lambda / 2] \cdot\left[R_{-a}\right] \cdot \vec{P}
$$

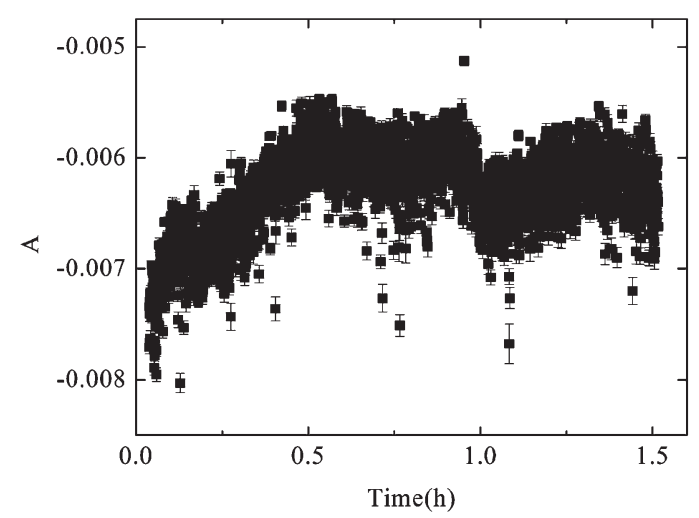

Figure 3. Long-term behavior of asymmetry without feedback. 
The polarizing beam splitters may be regarded as fixed LPs. The intensity asymmetry is therefore given by $P_{\text {final, } 1}=P_{\text {fi- }}$ nal, $0=\sin (4 a)$. Since $P_{\text {final, } 0}$ is defined to be 1 , we can simply extract the $P_{\text {final, } 1}$ component to obtain the asymmetry. The goal is to maintain the asymmetry at $A=0$; the magnitude of the rate of asymmetry change versus half-waveplate angle at these points is $4 / \mathrm{rad}$. If we wish to control the asymmetry to within $10^{-5}$, we must control the angle of the half-waveplate to $\sim 0.5$ arc sec. Since this is near the precision limit of commercially available nanorotators, the plane of polarization is instead fine-tuned electronically by changing the retardance of the LCR (see Figure 1).

We represent the LCR as a retarder with variable retardance $d$ that depends on a voltage $v$ :

$$
[\mathrm{LCR}]=\left[\begin{array}{rrrr}
1 & 0 & 0 & 0 \\
0 & 1 & 0 & 0 \\
0 & 0 & \cos (d(v)) & \sin (d(v)) \\
0 & 0 & -\sin (d(v)) & \cos (d(v))
\end{array}\right]
$$

The fast axis of this retarder is fixed at $45^{\circ}$. Therefore the final Stokes vector is given by $\vec{P}_{\text {final }}=\left[R_{a}\right] \cdot[\lambda / 2] \cdot\left[R_{-a}\right] \cdot$ $\left[R_{45^{\circ}}\right] \cdot[\mathrm{LCR}] \cdot\left[R_{-45^{\circ}}\right] \cdot P$. The resulting asymmetry is $A=$ $\cos (4 a) \cos (d(v))$.

The retardance-voltage curve provided by Meadowlark Optics is shown in Figure 4. Also shown in Figure 4 is our measurement of the LCR retardance-voltage curve obtained by placing the LCR between (imperfect) crossed polarizers and measuring the transmitted intensity as a function of $v$. Taking into account that the two polarizers have a value of $K_{\text {incident }}=0.965$ [15], we obtain the results of Figure 4 . Using these results and the Mueller analysis of the system in Figure 1, we can predict the optical system asymmetry as a function of LCR voltage. Both the predicted and the measured results of this are shown in Figure 5, where the half-waveplate angle is $0^{\circ}$ (vertical).

In a typical linear feedback scheme, we would measure the asymmetry and alter the system (i.e., output a voltage to the LCR) in a way that would make the asymmetry tend to zero. This would all take place during a discrete time

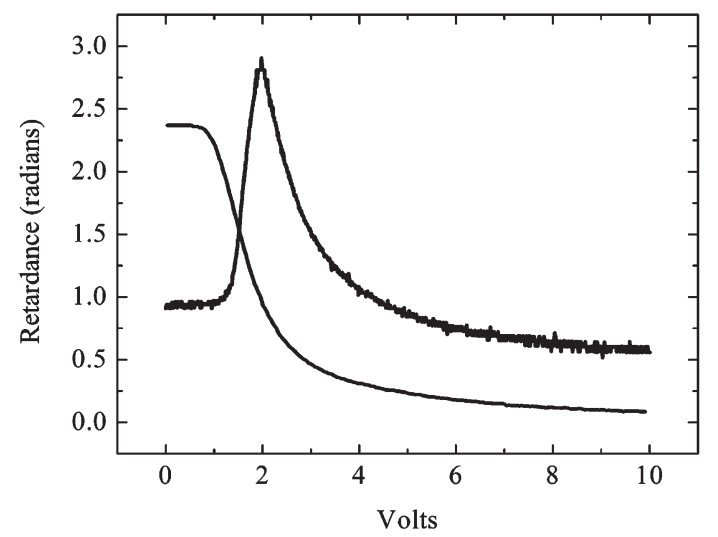

Figure 4. Retardance of LCR versus applied voltage: data provided by the manufacturer (monotonically decreasing curve) and measured value of this work (curve with peak).

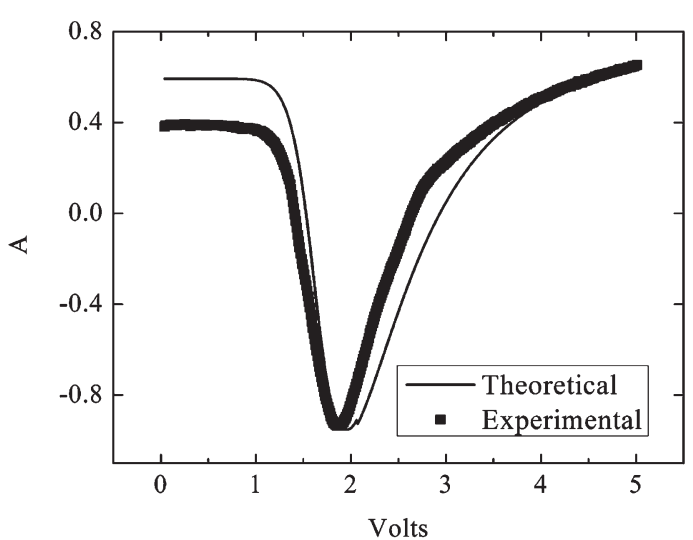

Figure 5. Asymmetry versus LCR voltage.

interval $i$. Our feedback voltage was determined by $v_{i+1}=$ $A_{i} k+v_{i}$, where $A_{i}$ is the measured asymmetry and $v_{i}$ is the voltage outputted during this time step. In the region near $A\left(v_{0}\right) \equiv 0, A(v)=\left.(\mathrm{d} A / \mathrm{d} v)\right|_{v_{0}}\left(v-v_{0}\right)$. Thus, for small $A, k=$ $-\mathrm{d} v /\left.\mathrm{d} A\right|_{v_{0}}$.

The asymmetry measurement must be made by averaging over many chopper cycles to avoid feeding back on noise. However, in the beginning of a feedback cycle, when changes in asymmetry are large, we average over fewer cycles so that the time it takes to feed back is not much larger than the time it takes for the asymmetry to change. We therefore use an adaptive method that alters the number of chopper cycles used to make a measurement with regard to how large the asymmetry is. We use only two categories of chopper cycle integration; if the asymmetry is $>0.001$, we use 10 chopper cycles, but if it is $<0.001$, we use 100 . We find that this is sufficient to feed back in a reasonable amount of time. Figure 6 shows the short-term behavior of the asymmetry when this feedback system is applied.

\section{Results}

Figure 7 shows the long-term asymmetry behavior with feedback, excluding the first few minutes when the asymmetry was $>10^{-4}$. As discussed in Section 3, when no feedback is applied, the asymmetry exhibits significant drifting

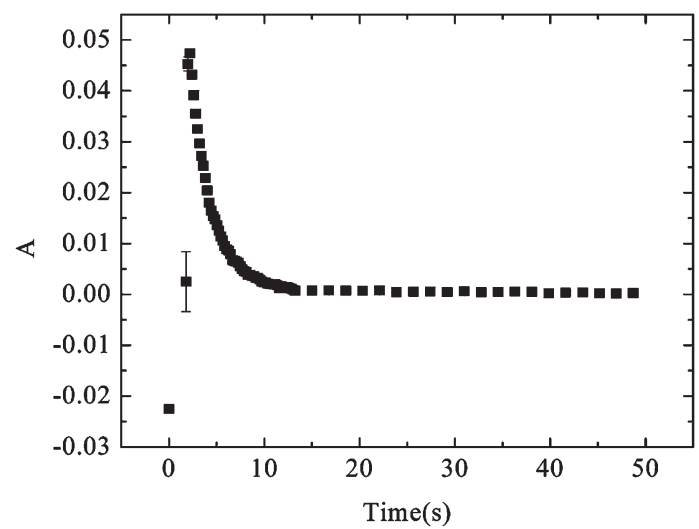

Figure 6. Short-term feedback behavior. 


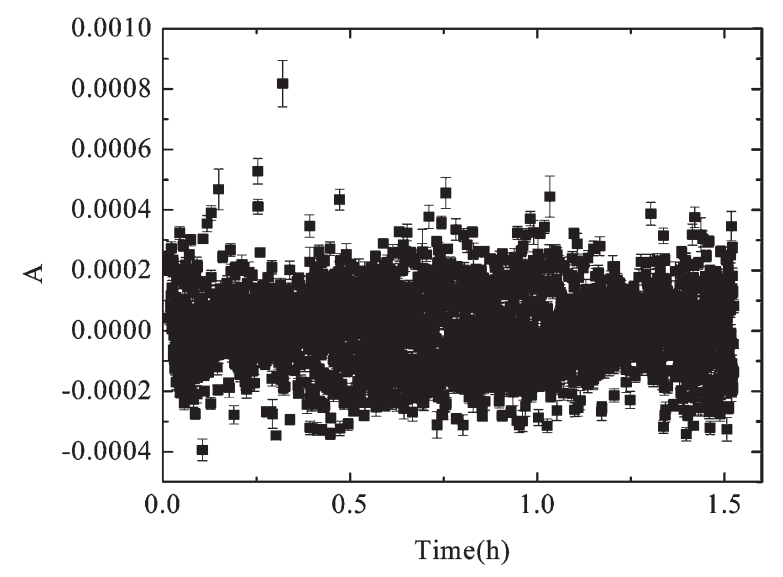

Figure 7. Long-term behavior of asymmetry with feedback.

to the point where it would eclipse any expected chiral effect (see Figure 3). Using this method we are able to maintain the asymmetry below $3 \times 10^{-4}$. Over $1.5 \mathrm{~h}$ the accumulated asymmetry of Figure 7 is $3: 7 \times 10^{-6} \pm 2.2 \times 10^{-6}$, well below the known values of ECD asymmetries [11]. In earlier results with a factor of 3 , worse LCR control voltage resolution limited the ability of this feedback method to control short-term asymmetries to better than $\sim 10^{-3}$ and could only achieve average asymmetry values below $1.5 \times$ $10^{-5}$ in periods of time greater than $15 \mathrm{~h}$.

\section{Conclusions}

We have demonstrated the ability to obtain long-term optical asymmetries well below $10^{-5}$. Our system has the advantage that helicity-correlated spatial variations of the beam are not caused by the optical elements responsible for helicity reversal. Active feedback to eliminate temporal drifting of asymmetry can thus be accomplished by elements not operating at the helicity-reversal frequency. We believe that the improved resolution of the LCR voltage control can improve even further the upper limit on shortterm asymmetry values, meaning that the upper limit on average asymmetry required by a given experiment can be reached in shorter and shorter periods of time. This optical system is ideally suited for producing GaAs photocurrent since it minimizes the drift of the laser beam over the surface of the crystal. In addition the feedback system described in this paper may be applied in a similar fashion to photocurrent, thereby eliminating any remaining helicity asymmetry.

\section{Acknowledgments}

We thank Jack Maseberg, Josh Machacek, and Steve Ducharme for helpful discussions. This work was supported by National Science Foundation grant PHY- 0653379 and Undergraduate Creative Activities and Research Experiences Research Funding through the University of Nebraska.

\section{References}

1. D. T. Pierce, "Spin-polarized electron sources," in Experimental Methods in the Physical Sciences-Atomic, Molecular, and Optical Physics: Atoms and Molecules, F. B. Dunning and R. G. Hulet, eds. (Academic, 1995), Vol. 29A, pp. 1-38.

2. A. S. Green, "Spin-dependent electron-molecule scattering," Ph.D. dissertation (University of Nebraska, 2004).

3. J. A. Zohner, "Reduction of instrumental asymmetries from an optics train," M.S. thesis (University of Nebraska, 2005).

4. T. Averett, C. E. Jones, R. D. McKeown, and M. Pitt, "Electron beam position stabilization with a piezo-electric optical correction system," Nucl. Instrum. Methods Phys. Res. A 438, 246-252 (1999).

5. T. B. Humensky, R. Alley, A. Brachmann, M. J. Browne, G. D. Cates, J. Clendenin, J. deLamare, J. Frisch, T. Galetto, E. W. Hughes, K. S. Kumar, P. Mastromarino, J. Sodja, P. A. Souder, J. Turner, and M. Woods, "SLAC's polarized electron source laser system and minimization of electron beam helicity correlations for the E-158 parity violation experiment," Nucl. Instrum. Methods Phys. Res. A 521, 261-298 (2004).

6. D. T. Spayde (SAMPLE Collaboration), "Updated results from the SAMPLE experiment," Euro. Phys. J. A 24, 51-54 (2005). See also D. T. Spayde, T. Averett, D. Barkhuff, D. H. Beck, E. J. Beise, C. Benson, H. Breuer, R. Carr, S. Covrig, J. DelCorso, G. Dodson, K. Dow, C. Eppstein, M. Farkhondeh, B. W. Filippone, P. Frazier, R. Hasty, T. M. Ito, C. E. Jones, W. Korsch, S. Kowalski, P. Lee, E. Maneva, K. McCarty, R. D. McKeown, J. Mikell, B. Mueller, P. Naik, M. Pitt, J. Ritter, V. Savu, M. Sullivan, R. Tieulent, E. Tsentalovich, S. P. Wells, B. Ang, and T. Zwart, "Parity violation in elastic electron-proton scattering and the proton's strange magnetic form factor," Phys. Rev. Lett. 84, 11061109 (2000).

7. M. Farkhondeh, W. Franklin, E. Tsentalovich, and T. Zwart, "Helicity-correlated effects in the SAMPLE experiment," AIP Conf. Proc. 675, 1024-1028 (2003).

8. J. Grames, P. Adderly, M. Baylac, J. Clark, A. Day, J. Hansknecht, M. Poelker, and M. Stutzman, "Status of the Jefferson Lab Polarized Beam Physics Program and preparations for upcoming parity experiments," AIP Conf. Proc. $675,1047-1052$ (2003).

9. K. W. Trantham, M. E. Johnston, and T. J. Gay, "Failure to observe electron circular dichroism in camphor," J. Phys. B 28, L543-L548 (1995).

10. T. J. Gay, M. E. Johnston, K. W. Trantham, and G. A. Gallup, "Scattering of chiral electrons by chiral molecules," in Selected Topics in Electron Physics, H. Kleinpoppen and M. C. Campbell, eds. (Plenum, 1996), pp. 159-170.

11. S. Mayer and J. Kessler, "Experimental verification of electron optic dichroism," Phys. Rev. Lett. 74, 4803-4806 (1995).

12. Hinds Instruments Model PEM-90.

13. T. C. Oakberg, "Modulated interference effects: use of photoelastic modulators with lasers," Opt. Eng. 34, 1545- 1550 (1995).

14. R. M. A. Azzam and N. M. Bashara, Ellipsometry and Polarized Light (North Holland, 1989).

15. H. G. Berry, G. Gabrielse, and A. E. Livingston, "Measurement of the Stokes parameters of light," Appl. Opt. 16, 3200- 3205 (1977). 